\title{
Selective Drivers of Simple Multicellularity
}

\author{
Kai Tong ${ }^{1,2, *}$, G. Ozan Bozdag ${ }^{1, *}$ and William C. Ratcliff ${ }^{1}$
}

*These authors contributed equally

Corresponding author: william.ratcliff@biology.gatech.edu

${ }^{1}$ School of Biological Sciences, Georgia Institute of Technology, Atlanta, GA, USA

${ }^{2}$ Interdisciplinary Graduate Program in Quantitative Biosciences, Georgia Institute of Technology, Atlanta, GA, USA

Highlights

- We survey ten key selective drivers favouring the evolution of simple multicellularity.

- The selective drivers of multicellularity depend on both lineage and environment.

- These drivers enhance self-protection, resource acquisition/production, or dispersal.

Keywords: Complexity; Evolution; Major transitions; Multicellularity; Selective driver;

Environment; Size; Division of labor

\section{ABSTRACT}

In order to understand the evolution of multicellularity, we must understand how and why selection favors the first steps in this process: the evolution of simple multicellular groups. Multicellularity has evolved many times in independent lineages with fundamentally different ecologies, yet no work has yet systematically examined these diverse selective drivers. Here we review recent developments in systematics, comparative biology, paleontology, synthetic biology, theory, and experimental evolution, highlighting ten selective drivers of simple multicellularity. Our survey highlights the many ecological opportunities available for simple multicellularity, and stresses the need for additional work examining how these first steps impact the subsequent evolution of complex multicellularity. 


\section{INTRODUCTION}

Multicellularity has evolved numerous times in distinct lineages [1]. From simple beginnings of small, simple clusters of cells, multicellular organisms have evolved spectacular, diverse forms, underpinning the evolution of novel ecosystems (e.g., forests, grasslands, and coral reefs) and transforming life on Earth. To understand how multicellularity evolves, we must understand how its initial steps, the evolution of simple multicellular groups, occur.

The vast majority of unicellular linages never evolve to be multicellular, presumably because the potential benefits of multicellularity do not outweigh its costs (i.e., nutrient / oxygen diffusion limitation, etc). Yet multicellularity has evolved many times in distinct lineages inhabiting markedly different ecologies. In this review, our goal is to describe key selective drivers of simple multicellularity, including both those underpinning the origin of multicellular group formation itself, and traits made possible by a multicellular life cycle (Figure 1).

In this review we focus on "simple" (in quotes, because this is a subjective classification, though here we use the heuristic of few cell types [2]) multicellular groups because it is a crucial stage of multicellular evolution: most multicellular lineages never evolve to be complex, and those that do necessarily started out simple. These multicellular groups are quite diverse, form facultatively or obligately, clonally or aggregatively, and may have no or limited cell differentiation. These drivers are presented in an order that roughly reflects whether one may favor the origin of group formation or drive group adaptation (often requiring some sort of cell-cell coordination or differentiation), although some drivers may impact multiple stages of multicellular evolution including complex multicellularity. How these drivers act in a specific lineage depends on its organismal and ecological context, yet some drivers may be more universal (e.g., predation avoidance and stress resistance), while others tend to be more lineage-specific (e.g., production and dispersal of propagules). This 
review is not intended to be exhaustive; indeed, this field is rapidly developing, and we anticipate that future discoveries will add to this body of work.

\section{EVOLUTIONARY DRIVERS OF SIMPLE MULTICELLULARITY}

\section{Predation avoidance}

Predation of unicellular microbes by protozoan or metazoan predators creates a strong selective pressure for traits that enhance their survival. A simple but effective defensive strategy is to form cellular groups that are too large to be eaten by the predator. Indeed, many unicellular microbes (e.g., algae and bacteria) facultatively form predation-resistant multicellular groups when predators are present (often by sensing predator-secreted chemicals), while others constitutively express simple multicellular forms [3-5]. These multicellular structures vary in morphology (e.g., filaments, sheets, spheroids, irregular clumps) and mode of group formation (i.e., incomplete cell separation, aggregation, or both) $[3,4,6,7]$. Continuously co-culturing unicellular algae [8-10] or fungi [11] with a predator has been shown to drive the evolution of simple multicellularity within weeks to months, which persisted even when the predators were removed. Paleontological records and phylogenetic analyses suggest that the evolution of unicellular (and later animal) predators in the Neoproterozoic may be a powerful driver of the origin and/or subsequent evolution of multicellularity in animals, green algae and red algae $[12,13]$. However, larger predators $[6,14]$ and raptorial protozoan predators [15] may in fact prefer colonial prey, thus the effect of group formation against predation may depend highly on the predator composition in the surrounding environment.

\section{Stress resistance}

Group formation is a widely-evolved strategy for dealing with environmental stress, as groups provide shelter for internal cells. In the yeast Saccharomyces cerevisiae, flocs (formed aggregatively) [16], lab-evolved snowflake-like clusters (formed clonally) [17] and small globose clumps in a wild strain (formed clonally) [18] all survived better under chemical 
stresses than single cells, and multicellular clusters also evolved under lab adaptation to low $\mathrm{pH}$ (in lactic acid but not in hydrochloric acid) [19]. This benefit may stem from physical shielding via diffusion limitation, and/or physiological changes of constituent (especially internal) cells that increase stress resistance [16]. Similarly, the unicellular green alga Chlamydomonas reinhardtii forms small palmelloid colonies or large aggregates when exposed to nutrient deficiency, salt stress, low or high $\mathrm{pH}$, oxidative stress, heat shock, etc., with larger groups being more stress-resistant [20-22]. Moreover, forming biofilms (surfaceattached aggregates of microbes) is a widespread multicellular solution across the unicellular tree of life to withstand various physical, chemical and biotic stressors, often mediated by concerted actions of multiple underlying mechanisms ([23,24]). In aggregative multicellular organisms (e.g., the cellular slime mould Dictyostelium discoideum and the myxobacterium Myxococcus xanthus), cells typically feed and proliferate in the unicellular stage, and actively aggregate when stressed to form multicellular fruiting bodies that contain aerially-lifted spores or cysts $[25,26]$. It is hypothesized that active aggregation into simple cell mounds may evolve prior to fruiting bodies, likely driven by its rapidly-conferred sheltering benefits against stresses [27].

\section{Improved extracellular metabolism}

Group formation may increase the efficiency of extracellular metabolism by both increasing the local concentration of extracellular metabolites and reducing their loss due to diffusion. Cells in a group may often be closely related (i.e., if the group develops clonally, or if aggregation occurs in a structured environment), limiting exploitation by non-productive cheats. Koschwanez and colleagues examined this using the yeast invertase system [28], a well-studied model of microbial cooperation [29]. In yeast, the cell-wall localized invertase enzyme breaks down extracellular sucrose into glucose and fructose. However, invertaseproducing cells cannot capture a significant fraction of the glucose and fructose as they diffuse away from the focal cell [30], inhibiting growth on low sucrose in diffusely populated unicellular populations [28]. When Koschwanez et al. [31] experimentally evolved unicellular 
yeast in low sucrose for 35 days, all replicate populations evolved into undifferentiated clonal multicellular groups that were able to grow in this low-resource environment by increasing the extracellular glucose concentration to sufficiently high levels. Furthermore, multicellular yeast efficiently privatized these extracellular common goods, preventing the invasion of non-producing cheats, whereas unicellular yeast performed poorly against cheats. Since extracellular enzymes predate multicellularity in fungi [32] and are widespread across microbes [33], including aggregative and filamentous species of multicellular bacteria $[34,35]$, selection for efficient extracellular metabolism may be a common route to multicellularity.

\section{Faster sedimentation}

By Stokes' law, organisms sink faster with increased size and density. Group formation increases size and thus sedimentation rate, allowing aquatic organisms to reach deeper positions in the water column to access different nutrients or evade predators. A recent study showed that in the ichthyosporean Sphaeroforma genus (close unicellular relatives of animals, with transient multicellular stage), sedimentation rate is a variable and evolvable trait affected by multicellular size and density, which may be used to control the position of these immotile organisms in the water column [36]. In particular, continuously selecting Sphaeroforma arctica in the lab for fast settling through liquid media led to the evolution of fast-settling clonal clumps [36]. Similar settling selection has also been used to evolve multicellularity and increased size in the unicellular yeast S. cerevisiae $[37,38]$ (even macroscopic size [39]) and Kluyveromyces lactis [40] and the unicellular alga C. reinhardtii [41]. Fast-sedimenting phenotypes also rapidly evolve in human-altered ecosystems, like water-treatment plants [42]. However, faster sedimentation due to group formation is often considered as a cost to phytoplankton, as it reduces their access to light $[3,6]$. This cost is countered in some species by mobility or buoyancy control, and is alleviated in shallow or mixed water bodies $[3,6,43]$. 


\section{Increased motility}

Forming cooperative groups is a powerful mechanism to increase organismal motility, which allows organisms to move more rapidly towards favorable environments and away from dangers. Multicellular $D$. discoideum slugs migrate faster than solitary cells, with larger slugs capable of achieving higher speeds, and are able to cross soil barriers that solitary cells cannot $[44,45]$. In the volvocine algae, colonial species (e.g., Volvox carteri) swim faster than their unicellular relative C. reinhardtii (also in a size-dependent manner), enabling more sustained access to light and nutrients $[43,46]$. Interestingly, the sessile stalked cells of the bacterium Caulobacter crescentus can become motile by forming aggregates where flagellated cells are intermittently produced through asymmetric cell division and propel the aggregate to roll on the surface [47]. However, group formation alone may not be sufficient to confer increased motility. Predation/stress-induced and lab-evolved colonial forms of $C$. reinhardtii have impaired motility due to lost, excised, or internally oriented flagella [48-50]. While $V$. carteri beats flagella in a coordinated direction [46], facultative rosette colonies in the choanoflagellate Salpingoeca rosetta exhibit uncoordinated flagellar motion [51,52], which lead to flagellar forces largely cancelling out each other and thus slower swimming speed than single cells [15]. Finally, multicellular configuration may also affect organismal motility. For example, in the choanoflagellate Choanoeca flexa, the cup-shaped colonies can respond to light-to-dark transition by rapidly inverting their curvature with flagella changing from pointing inwards to outwards, which results in enhanced swimming and reduced feeding [53].

\section{Benefits of chimerism}

While clonal multicellular organisms typically develop from a single cell and thereby minimize within-group genetic heterogeneity, aggregative multicellular organisms form via the coming together of potentially-unrelated cells. Indeed, genetically chimeric fruiting bodies of the aggregative organisms $D$. discoideum [54] and $M$. xanthus [55-57] are frequently found in the wild and readily formed in the lab. Chimerism has long been known to impose costs on 
group fitness (chimeric load), often in the form of genetic conflict (e.g., cheating) $[1,58]$. However, a recent study found that wild $M$. xanthus clones isolated from the same fruiting body often produce more spores in chimeric groups than each alone, suggesting synergistic interactions among genotypes that improve group performance (chimeric synergy) [56]. Genome sequencing indicated that such chimeric synergy may be the result of group-level selection promoting synergistic coevolution of diversified kin lineages that remain together and repeatedly interact over long periods [57]. Another benefit of chimerism may be ecological: low barriers to group formation should, in principle, increase the rate at which groups form and/or their final size, potentially providing size-related advantages. For example, given the same cell number per clone, chimeric slugs of $D$. discoideum are larger than monoclonal ones and thus can migrate farther, despite the presence of chimeric load [59].

\section{Cross-feeding and division of labor}

Interactions among single- or multi-species communities provide ample opportunity for metabolic complementarity and differentiation, and can drive the evolution of novel higherlevel organisms [60,61]. For example, cross-feeding in bacterial multicellular communities can increase fitness by raising resource utilization efficiency and growth rate [62-64]. This benefit arises because uni- or bi-directional resource sharing allows communities to increase their metabolic efficiency.

Cross-feeding readily evolves in single-species bacterial colonies or biofilms because direct physical contact between cells enables more efficient metabolite sharing [65]. Furthermore, compact, three-dimensional growth creates diffusion gradients, such as oxic and anoxic zones [66], regulating the formation of distinct cell populations running complementary biochemical pathways [67]. For instance, in single-species communities of Escherichia coli or Pseudomonas aeruginosa, metabolite sharing can be advantageous due to an increased 
carbon consumption efficiency $[63,68,69]$. Recent work has also showed that cooperative cross-feeding can readily evolve in multi-species communities [64].

Following the formation of simple multicellular groups, the evolution of division of labor can increase the efficiency of resource utilization and production $[70,71]$ without inferring taskswitching costs [72]. While unicellular organisms may perform two vital yet incompatible processes by switching between them in time, multicellularity allows tasks to be segregated across cells in space [1]. Two classical examples are the division of labor between photosynthetic cells and nitrogen-fixing heterocysts in filamentous cyanobacteria [73], and differentiation into flagellated somatic cells and non-flagellated reproductive germ cells in colonial volvocine algae $[43,71,74]$. There are trade-offs in both cases that limit individual cells from performing both tasks simultaneously, in the former example nitrogen-fixing enzymes must work in an oxygen-depleted condition not compatible with oxygenic photosynthesis, and in the latter the microtubule-organizing center cannot be used simultaneously for flagellar motility and mitosis. Interestingly, a similar motility-mitosis tradeoff may have played a role in the origin of animals $[75,76]$. Reconstructing the order of trait emergence indeed suggests that nitrogen fixation may be a prime driver of cyanobacterial multicellularity [77]. More broadly, stress-induced differentiation into spores and lineagespecific supportive cell types is widespread in microorganisms (discussed further below). Moreover, some constituent cells can even be sacrificed through programmed cell death to benefit the group by providing extra nutrients [78-80], forming multicellular structures (e.g., stalk for raising spores [81]), or fragmenting the group to release propagules $[37,73]$.

Other examples of microbial division of labor have been reviewed elsewhere $[70,82-85]$ and novel types of division of labor are frequently discovered (see for instance $[86,87])$. Notably, recent work has shown that division of labor can arise via a single mutation or gene $[88,89]$, and that the topology of simple multicellular bodies strongly favors germ-soma cellular differentiation [90]. Taken together, this work suggests that division of labor may either be a 
driver of group formation (in the case of cross-feeding), or evolve shortly after group formation, providing selective benefits even in the simplest multicellular groups.

\section{Competitive overgrowth}

Multicellularity can allow surface-attached organisms to overgrow competitors and thus gain preferential access to exogenous resources. For example, crust-forming algae are the first to colonize bare rock, but are subsequently overgrown by filamentous, turf-forming multicellular taxa [91]. The arms race for access to light above ground, and nutrients and water below ground, have long been thought to be a major driver of land plant evolution (though other drivers, like increased propagule dispersal with height, may have played an important role in tree evolution as well [92]). Similarly, microbes growing on a solid substrate may gain an advantage by overgrowing competitors [93], resulting in structural similarities between millimeter-scale biofilms and hundreds-of-meters-scale forests [94].

\section{Efficient utilization of patchy resources}

Multicellularity can provide an advantage in patchy resource landscapes. The most striking example of this is the hyphal multicellular fungi, which can transport nutrients from resourcepoor to resource-rich environments through their cytoplasmic networks [95]. Filamentous multicellularity provides an advantage because it allows for nutritional arbitrage in heterogeneous, unmixed landscapes. Immotile unicellular heterotrophs will stop growing when they run out of a locally-available limiting resource, even if they have a surplus of all other essential resources. But a multicellular organism capable of transporting resources among its cells can leverage the power of cell-cell trade to continue growing, even when locally-available resources would not support it [96]. While this has been taken to an extreme in modern fungi, the benefits of nutrient transport doubtless act over the smaller scales of nascent multicellular organisms as well. Filamentous growth is not solely the domain of fungi. For example, primitive embryophytes (early multicellular forms in plants) also formed branched, filamentous bodies, which have been hypothesized to be important for exploring 
local resource environments $[97,98]$. More generally, patchy resources are thought to be a key driver of evolutionary transitions in individuality across scales, including the evolution of "super organismality" in both insects and mammals [99,100].

Within poorly-mixed liquid environments, multicellularity can also facilitate the capture of patchy resources. For example, collective flagellar motions can stir and mix the surrounding fluid, improving the efficiency of nutrient uptake in colonial volvocine algae $[46,101]$ and prey capture in some colonial choanoflagellates [15].

\section{Production and dispersal of propagules}

In the lineages known to aggregate and form spore-laden fruiting bodies, multicellular development may also aid in the production and dispersal of stress-resistant propagules (also see spore formation in the bacterium Bacillus subtilis and the actinomycete Streptomyces coelicolor [102]). Spores can withstand harsh environmental conditions $[102,103]$, in part because some components released by dying cells during fruiting body development may be incorporated to build resilient spore coats $[27,80,104]$. Indeed, frost resistance was recently proposed as a potential cause of multicellular sporulation in Dictyostelia, as an adaptation to global cooling in the Neoproterozoic [104]. Moreover, fruiting bodies can further protect spores by shielding them (e.g., in a protective extracellular matrix [105]) and lifting them above hazards of the soil $[81,106]$.

Fruiting bodies have also long been hypothesized to enhance spore dispersal by lifting spores in the air to better contact passing animals, air currents, or water $[27,106,107]$. Lab studies found that fruit flies can acquire more spores from intact fruiting bodies of $D$. discoideum than from disrupted ones [108]. However, this dispersal hypothesis lacks direct tests of whether fruiting bodies can indeed facilitate dispersing spores to favorable environments, and it does not explain the morphological diversity of fruiting bodies [27]. Notably, in a subgroup of Dictyostelia (including D. discoideum), aggregation initially forms 
motile multicellular slugs [26], which may also improve spore dispersal by migrating to suitable environments before transforming into fruiting bodies [44,109]. Finally, it has also been hypothesized that concentrating spores in fruiting bodies may be beneficial in $M$. xanthus because high spore density facilitates germination and cooperative feeding when encountering favorable environments [110].

\section{CONCLUSION}

Simple multicellularity has evolved numerous times across the tree of life and takes a diverse array of forms. In this mini review we described ten key selective drivers for simple multicellularity, all of which stem from the benefits of increased size or novel cell-cell interactions, and serve as a collective solution to self-protection, resource acquisition/production, or improved dispersal. While multicellularity has evolved numerous times, given the geological time scales at play, it is still a relatively rare innovation and the vast majority of unicellular lineages have stayed unicellular. This may be because multicellular growth imposes significant costs (e.g., nutrient and oxygen diffusion limitation, impaired motility, reduced passive dispersal in air or water currents, metabolic costs required for group formation and maintenance, the potential for social exploitation, vulnerability to predators or pathogens, etc.) which must be overcome by a multicellular-specific benefit. Yet it is clear from the examples described above that there are many routes to multicellularity, with each route being highly dependent on the nature of the organism (e.g., feeding mode, motility, mode of group formation, multicellular configuration, constraints of cell biology) and the spatiotemporal dynamics of its environment.

We chose to examine the selective drivers of simple multicellularity because it is both important and relatively understudied. Indeed, it remains challenging to determine the selective benefit of nascent multicellularity in extant multicellular lineages, in part because the early evolution of multicellularity is often difficult to resolve. While we may never have certainty about how specific lineages first evolved multicellularity, advances in phylogenetics 
/ comparative biology (e.g., [77,85]), paleontology (e.g., [12,104]), synthetic biology (e.g., $[39,111])$, theory (e.g., [96]), experimental evolution (e.g., [39,112]), and experiments with non-model organisms (e.g., $[36,53])$ will continue to refine our hypotheses. We see great value in this approach for understanding the evolution of multicellularity, helping address a number of outstanding questions: $i)$ How, when, and why do simple multicellular organisms evolve from unicellular ancestors, especially in their ecological contexts? ii) How do the dynamics (traits, mechanisms, etc.) of a nascent multicellular lineage influence the subsequent evolution of multicellular complexity? iii) Which ecological drivers of multicellularity stabilize with relatively small, simple organisms, and which result in an openended arms race? iv) Under what conditions do evolutionary priority effects constrain subsequent innovation, and how does the breadth of multicellular niches affect the number of independent transitions to multicellularity within different clades? Both recent developments in technology (i.e., synthetic biology, genome editing, genome and single-cell sequencing, etc.) and extensive collaboration among researchers with diverse backgrounds make this a particularly exciting time to work on the evolution of multicellularity.

Figure 1. Ten selective drivers of simple multicellularity. 
1. Predation avoidance
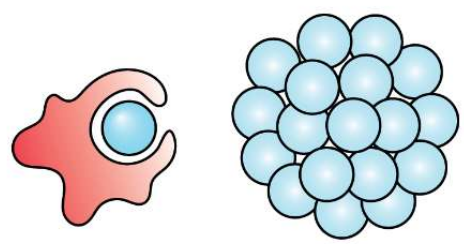

3. Improved extracellular metabolism

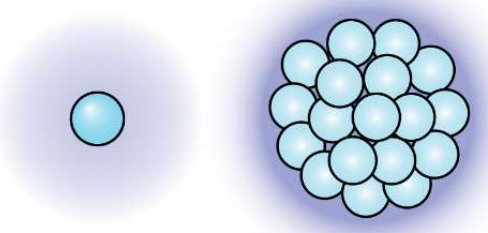

5. Increased motility

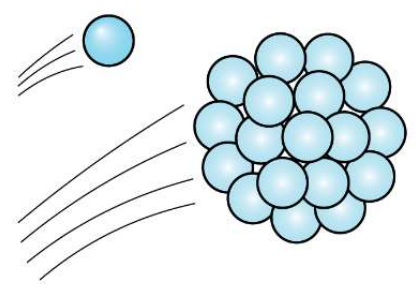

7. Cross-feeding and division of labor

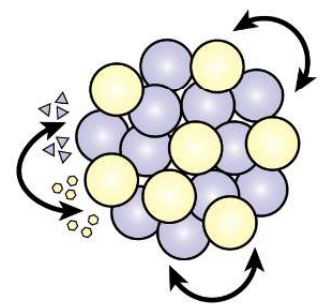

9. Efficient utilization of patchy resources

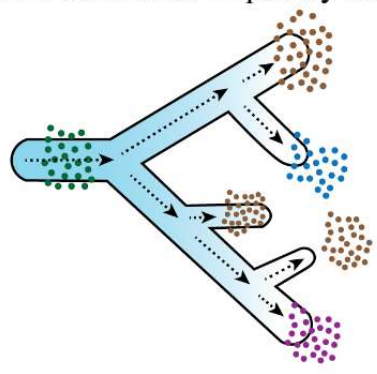

2. Stress resistance

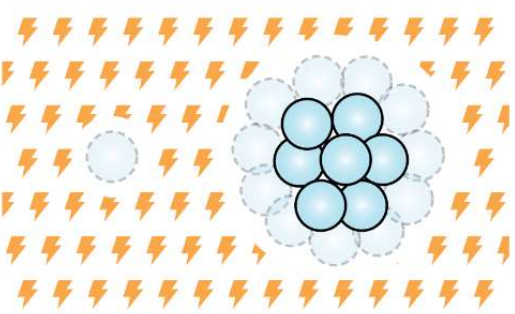

4. Faster sedimentation

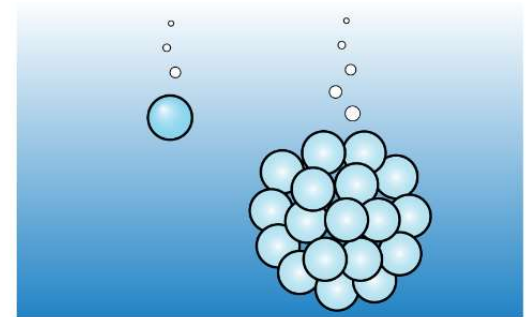

6. Benefits of chimerism

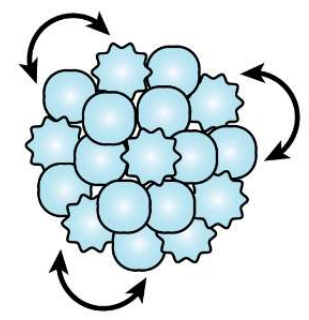

8. Competitive overgrowth

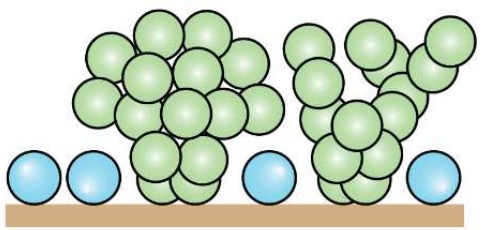

10. Production and dispersal of propagules

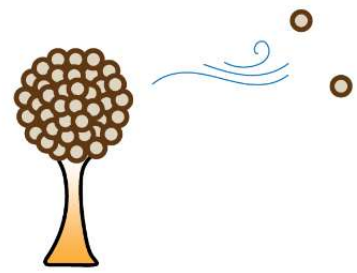

\section{ACKNOWLEDGEMENTS}


We thank Matt Herron and all members of the Ratcliff group for insightful comments on the manuscript. This work was supported by NSF grant DEB-1845363 and Packard Fellowship for Science and Engineering to W.C. Ratcliff.

\section{HIGHLIGHTED PAPERS}

*Kapsetakis et al. 2019: This study investigates the benefits and costs of multicellular formation in algae. In particular, it uses experiments and meta-analysis to show that multicellular groups can still be susceptible to larger predators, and slower growth rate of multicellular groups often manifest only under nutrient-limiting conditions.

**Koehl 2020: This review presents a comprehensive synthesis of benefits and costs of multicellularity in choanoflagellates, the closest living relatives of animals.

*Fortezza et al. 2021: This review critically synthesizes and proposes selective factors that drive each of three life cycle phases in the aggregative multicellular lineages, highlighting Dictyostelia and Myxococcales.

*Lawal et al. 2020: This study proposes cold climate adaptation as a new hypothesis for the origin of multicellular sporulation in Dictyostelia, by bringing together experimental, biogeographical, paleontological and phylogenetic evidence.

*Brunet et al. 2019: The authors discovered a novel choanoflagellate species, Choanoeca flexa, which can invert its multicellular curvature upon a light-to-dark transition, and they investigated its ecological consequences, molecular mechanisms and evolutionary implications.

*Hammerschmidt et al. 2021: This study reconstructs the order of trait emergence in cyanobacteria, suggesting nitrogen fixation as the prime driver for cyanobacteria 
multicellularity, which was followed by the evolution of reproductive life cycle, differentiated cells and patterned multicellularity.

*Umen and Herron, 2021: This review summarizes the multicellularity in several groups of green algae, highlighting their life cycles, phylogenies, genetics of multicellular traits, and genetic and genomic resources.

*Wielgoss et al. 2019: This study shows that isolates within wild-caught myxobacteria fruiting bodies are diversified kin lineages with recurrent mutations in social genes that may be favored by group-level selection.

*Pande et al. 2016: In contrast to theoretical predictions, crossfeeding bacterial biofilms can resist the invasion of non-cooperating individuals by actively forming spatially structured communities, hinting that such cooperative interactions should be widespread in nature.

*Koschwanez et al. 2011: Examining the utilization of extracellular public goods in yeast, authors show that simple multicellular groups can grow in low carbon and fare better against cheats, whereas single-celled yeast cannot.

*Koschwanez et al. 2013: In this landmark evolution experiment, authors show that selection for improved use of extracellular public goods leads to the evolution of simple multicellularity from single-celled ancestors in yeast.

*Whiteside et al. 2019: By developing a quantum-dot particle tracking method, researchers show that fungi can redistribute resources across their multicellular network due to unequal resource distribution. 
*Heaton et al. 2020: This modeling study shows that transporting resources through cytoplasmic networks maximizes resource utilization efficiency and extends exoenzymes usage, providing an explanation for the origins of multicellularity in hyphal fungi.

*Dudin et al. 2021: Using the ichthyosporean Sphaeroforma genus, the authors show that sedimentation rate can be a highly variable and evolvable trait controlled by size and density, shaping the evolution of multicellularity.

*Bozdag et al. 2021: This study is a remarkable demonstration of the power of long-term experimental evolution in studying the origin of multicellularity, by evolving macroscopic multicellularity de novo and examining its physical basis.

\section{REFERENCES}

1. Grosberg RK, Strathmann RR: The evolution of multicellularity: a minor major transition? Annu. Rev. Ecol. Evol. Syst. 2007, 38:621-654.

2. Knoll AH: The multiple origins of complex multicellularity. Annual Review of Earth and Planetary Sciences 2011, 39:217-239.

3. Lürling M: Grazing resistance in phytoplankton. Hydrobiologia 2021, 848:237-249.

4. Jousset A: Ecological and evolutive implications of bacterial defences against predators. Environmental microbiology 2012, 14:1830-1843.

5. Kessin RH, Gundersen GG, Zaydfudim V, Grimson M: How cellular slime molds evade nematodes. Proceedings of the National Academy of Sciences 1996, 93:4857-4861.

6. Pančić M, Kiørboe T: Phytoplankton defence mechanisms: traits and trade - offs. Biological Reviews 2018, 93:1269-1303.

7. Kapsetaki SE, Tep A, West SA: How do algae form multicellular groups? Evolutionary Ecology Research 2017, 18:663-675.

8. Boraas ME, Seale DB, Boxhorn JE: Phagotrophy by a flagellate selects for colonial prey: a possible origin of multicellularity. Evolutionary Ecology 1998, 12:153-164.

9. Becks L, Ellner SP, Jones LE, Hairston Jr NG: Reduction of adaptive genetic diversity radically alters eco - evolutionary community dynamics. Ecology letters 2010, 13:989-997.

10. Herron MD, Borin JM, Boswell JC, Walker J, Chen I-CK, Knox CA, Boyd M, Rosenzweig F, Ratcliff WC: De novo origins of multicellularity in response to predation. Scientific reports 2019, 9:1-9.

11. Brunke S, Seider K, Fischer D, Jacobsen ID, Kasper L, Jablonowski N, Wartenberg A, Bader O, Enache-Angoulvant A, Schaller M: One small step for a yeastmicroevolution within macrophages renders Candida glabrata hypervirulent due to a single point mutation. PLoS pathogens 2014, 10:e1004478.

12. Knoll AH, Lahr D: Fossils, feeding, and the evolution of complex multicellularity. Multicellularity, Origins and Evolution, The Vienna Series in Theoretical Biology: Boston, Massachusetts Institute of Technology 2016:1-16. 
13. Stanley SM: An ecological theory for the sudden origin of multicellular life in the late Precambrian. Proceedings of the National Academy of Sciences 1973, 70:1486-1489.

14. Kapsetaki SE, West SA: The costs and benefits of multicellular group formation in algae. Evolution 2019, 73:1296-1308.

15. Koehl M: Selective factors in the evolution of multicellularity in choanoflagellates. Journal of Experimental Zoology Part B: Molecular and Developmental Evolution 2021, 336:315-326.

16. Smukalla S, Caldara M, Pochet N, Beauvais A, Guadagnini S, Yan C, Vinces MD, Jansen A, Prevost MC, Latgé J-P: FLO1 is a variable green beard gene that drives biofilm-like cooperation in budding yeast. Cell 2008, 135:726-737.

17. Alugoju P, Mahilkar A, Saini S: Evolution of multicellularity and unicellularity in yeast S. cerevisiae to study reversibility of evolutionary trajectories. bioRxiv 2021:2020.2008. 2015.252361.

18. Kuzdzal - Fick JJ, Chen L, Balázsi G: Disadvantages and benefits of evolved unicellularity versus multicellularity in budding yeast. Ecology and evolution 2019, 9:8509-8523.

19. Fletcher E, Feizi A, Bisschops MM, Hallström BM, Khoomrung S, Siewers V, Nielsen J: Evolutionary engineering reveals divergent paths when yeast is adapted to different acidic environments. Metabolic engineering 2017, 39:19-28.

20. de Carpentier F, Lemaire SD, Danon A: When unity is strength: the strategies used by Chlamydomonas to survive environmental stresses. Cells 2019, 8:1307.

21. Cheloni G, Slaveykova VI: Morphological plasticity in Chlamydomonas reinhardtii and acclimation to micropollutant stress. Aquatic Toxicology 2021, 231:105711.

22. de Carpentier F, Maes A, Marchand CH, Chung C, Durand C, Crozet P, Lemaire SD, Danon A: Stress-induced collective behavior leads to the formation of multicellular structures and the survival of the unicellular alga Chlamydomonas. bioRxiv 2021.

23. De la Fuente-Núñez C, Reffuveille F, Fernández L, Hancock RE: Bacterial biofilm development as a multicellular adaptation: antibiotic resistance and new therapeutic strategies. Current opinion in microbiology 2013, 16:580-589.

24. Van Acker H, Van Dijck P, Coenye T: Molecular mechanisms of antimicrobial tolerance and resistance in bacterial and fungal biofilms. Trends in microbiology 2014, 22:326-333.

25. Du Q, Kawabe Y, Schilde C, Chen Z-h, Schaap P: The evolution of aggregative multicellularity and cell-cell communication in the Dictyostelia. Journal of molecular biology 2015, 427:3722-3733.

26. Kawabe Y, Du Q, Schilde C, Schaap P: Evolution of multicellularity in Dictyostelia. The International journal of developmental biology 2019, 63:359.

27. La Fortezza M, Schaal K, Velicer GJ: Why aggregate? On the evolution of aggregative multicellularity. 2021.

28. H. Koschwanez J, R. Foster K, W. Murray A: Sucrose utilization in budding yeast as a model for the origin of undifferentiated multicellularity. PLoS biology 2011, 9:e1001122.

29. Bozdag G, Greig D: The genetics of a putative social trait in natural populations of yeast. Molecular ecology 2014, 23:5061-5071.

30. Gore J, Youk H, Van Oudenaarden A: Snowdrift game dynamics and facultative cheating in yeast. Nature 2009, 459:253-256.

31. Koschwanez JH, Foster KR, Murray AW: Improved use of a public good selects for the evolution of undifferentiated multicellularity. Elife 2013, 2:e00367.

32. Lange L, Pilgaard B, Herbst F-A, Busk PK, Gleason F, Pedersen AG: Origin of fungal biomass degrading enzymes: evolution, diversity and function of enzymes of early lineage fungi. Fungal Biology Reviews 2019, 33:82-97.

33. West SA, Diggle SP, Buckling A, Gardner A, Griffin AS: The social lives of microbes. Annu. Rev. Ecol. Evol. Syst. 2007, 38:53-77. 
34. Konovalova A, Petters T, Søgaard-Andersen L: Extracellular biology of Myxococcus xanthus. FEMS microbiology reviews 2010, 34:89-106.

35. Chater KF, Biró S, Lee KJ, Palmer T, Schrempf H: The complex extracellular biology of Streptomyces. FEMS microbiology reviews 2010, 34:171-198.

36. Dudin O, Wielgoss S, New AM, Ruiz-Trillo I: Regulation of sedimentation rate shapes the evolution of multicellularity in a unicellular relative of animals. bioRxiv 2021.

37. Ratcliff WC, Denison RF, Borrello M, Travisano M: Experimental evolution of multicellularity. Proceedings of the National Academy of Sciences 2012, 109:15951600.

38. Oud B, Guadalupe-Medina V, Nijkamp JF, de Ridder D, Pronk JT, van Maris AJ, Daran $\mathrm{J}-\mathrm{M}$ : Genome duplication and mutations in ACE2 cause multicellular, fastsedimenting phenotypes in evolved Saccharomyces cerevisiae. Proceedings of the National Academy of Sciences 2013, 110:E4223-E4231.

39. Bozdag GO, Zamani-Dahaj SA, Kahn PC, Day TC, Tong K, Balwani AH, Dyer EL, Yunker PJ, Ratcliff WC: De novo evolution of macroscopic multicellularity. bioRxiv 2021.

40. Driscoll WW, Travisano M: Synergistic cooperation promotes multicellular performance and unicellular free-rider persistence. Nature communications 2017, 8:1-10.

41. Ratcliff WC, Herron MD, Howell K, Pentz JT, Rosenzweig F, Travisano M: Experimental evolution of an alternating uni-and multicellular life cycle in Chlamydomonas reinhardtii. Nature communications 2013, 4:1-7.

42. Chaignon V, Lartiges B, El Samrani A, Mustin C: Evolution of size distribution and transfer of mineral particles between flocs in activated sludges: an insight into floc exchange dynamics. Water Research 2002, 36:676-684.

43. Solari CA, Kessler JO, Michod RE: A hydrodynamics approach to the evolution of multicellularity: flagellar motility and germ-soma differentiation in volvocalean green algae. The American Naturalist 2006, 167:537-554.

44. Kuzdzal-Fick JJ, Foster KR, Queller DC, Strassmann JE: Exploiting new terrain: an advantage to sociality in the slime mold Dictyostelium discoideum. Behavioral Ecology 2007, 18:433-437.

45. Bonner JT, Koontz Jr PG, Paton D: Size in relation to the rate of migration in the slime mold Dictyostelium discoideum. Mycologia 1953, 45:235-240.

46. Short MB, Solari CA, Ganguly S, Powers TR, Kessler JO, Goldstein RE: Flows driven by flagella of multicellular organisms enhance long-range molecular transport. Proceedings of the National Academy of Sciences 2006, 103:8315-8319.

47. Zeng Y, Liu B: Self-propelling and rolling of a sessile-motile aggregate of the bacterium Caulobacter crescentus. Communications biology 2020, 3:1-8.

48. Lurling M, Beekman W: Palmelloids formation in Chlamydomonas reinhardtii: defence against rotifer predators? In Annales de Limnologie-International Journal of Limnology: EDP Sciences: 2006:65-72.

49. Khona DK, Shirolikar SM, Gawde KK, Hom E, Deodhar MA, D'Souza JS:

Characterization of salt stress-induced palmelloids in the green alga, Chlamydomonas reinhardtii. Algal Research 2016, 16:434-448.

50. Boyd M, Rosenzweig F, Herron MD: Analysis of motility in multicellular Chlamydomonas reinhardtii evolved under predation. Plos one 2018, 13:e0192184.

51. Roper M, Dayel MJ, Pepper RE, Koehl M: Cooperatively generated stresslet flows supply fresh fluid to multicellular choanoflagellate colonies. Physical review letters 2013, 110:228104.

52. Kirkegaard JB, Marron AO, Goldstein RE: Motility of colonial choanoflagellates and the statistics of aggregate random walkers. Physical review letters 2016, 116:038102. 
53. Brunet T, Larson BT, Linden TA, Vermeij MJ, McDonald K, King N: Light-regulated collective contractility in a multicellular choanoflagellate. Science 2019, 366:326-334.

54. Gilbert OM, Foster KR, Mehdiabadi NJ, Strassmann JE, Queller DC: High relatedness maintains multicellular cooperation in a social amoeba by controlling cheater mutants. Proceedings of the National Academy of Sciences 2007, 104:8913-8917.

55. Kraemer SA, Velicer GJ: Endemic social diversity within natural kin groups of a cooperative bacterium. Proceedings of the National Academy of Sciences 2011, 108:10823-10830.

56. Pande S, Velicer GJ: Chimeric synergy in natural social groups of a cooperative microbe. Current Biology 2018, 28:262-267. e263.

57. Wielgoss S, Wolfensberger R, Sun L, Fiegna F, Velicer GJ: Social genes are selection hotspots in kin groups of a soil microbe. Science 2019, 363:1342-1345.

58. Rainey PB, De Monte S: Resolving conflicts during the evolutionary transition to multicellular life. Annual Review of Ecology, Evolution, and Systematics 2014, 45:599-620.

59. Foster KR, Fortunato A, Strassmann JE, Queller DC: The costs and benefits of being a chimera. Proceedings of the Royal Society of London. Series B: Biological Sciences 2002, 269:2357-2362.

60. D'Souza G, Kost C: Experimental evolution of metabolic dependency in bacteria. PLoS genetics 2016, 12:e1006364.

61. Libby E, Ratcliff WC: Lichens and microbial syntrophies offer models for an interdependent route to multicellularity. The Lichenologist 2021, 53:283-290.

62. Pfeiffer T, Bonhoeffer S: Evolution of cross-feeding in microbial populations. The American naturalist 2004, 163:E126-E135.

63. Cole JA, Kohler L, Hedhli J, Luthey-Schulten Z: Spatially-resolved metabolic cooperativity within dense bacterial colonies. BMC systems biology 2015, 9:1-17.

64. Pande S, Kaftan F, Lang S, Svatoš A, Germerodt S, Kost C: Privatization of cooperative benefits stabilizes mutualistic cross-feeding interactions in spatially structured environments. The ISME journal 2016, 10:1413-1423.

65. D'Souza G, Shitut S, Preussger D, Yousif G, Waschina S, Kost C: Ecology and evolution of metabolic cross-feeding interactions in bacteria. Natural Product Reports 2018, 35:455-488.

66. SchiessI KT, Hu F, Jo J, Nazia SZ, Wang B, Price-Whelan A, Min W, Dietrich LE: Phenazine production promotes antibiotic tolerance and metabolic heterogeneity in Pseudomonas aeruginosa biofilms. Nature communications 2019, 10:1-10.

67. Stewart PS, Franklin MJ: Physiological heterogeneity in biofilms. Nature Reviews Microbiology 2008, 6:199-210.

68. Wolfsberg E, Long CP, Antoniewicz MR: Metabolism in dense microbial colonies: $13 \mathrm{C}$ metabolic flux analysis of E. coli grown on agar identifies two distinct cell populations with acetate cross-feeding. Metabolic engineering 2018, 49:242-247.

69. Lin Y-C, Cornell WC, Jo J, Price-Whelan A, Dietrich LE: The Pseudomonas aeruginosa complement of lactate dehydrogenases enables use of $\mathrm{d}$-and Ilactate and metabolic cross-feeding. MBio 2018, 9:e00961-00918.

70. West SA, Cooper GA: Division of labour in microorganisms: an evolutionary perspective. Nature Reviews Microbiology 2016, 14:716-723.

71. Michod RE: Evolution of individuality during the transition from unicellular to multicellular life. Proceedings of the National Academy of Sciences 2007, 104:8613-8618.

72. Goldsby HJ, Dornhaus A, Kerr B, Ofria C: Task-switching costs promote the evolution of division of labor and shifts in individuality. Proceedings of the National Academy of Sciences 2012, 109:13686-13691.

73. Herrero A, Stavans J, Flores E: The multicellular nature of filamentous heterocystforming cyanobacteria. FEMS Microbiology Reviews 2016, 40:831-854. 
74. Koufopanou V: The evolution of soma in the Volvocales. The American Naturalist 1994, 143:907-931.

75. Buss LW: Evolution, development, and the units of selection. Proceedings of the National Academy of Sciences 1983, 80:1387-1391.

76. King N: The unicellular ancestry of animal development. Developmental cell 2004 , 7:313-325.

77. Hammerschmidt K, Landan G, Domingues Kümmel Tria F, Alcorta J, Dagan T: The order of trait emergence in the evolution of cyanobacterial multicellularity. Genome biology and evolution 2021, 13:evaa249.

78. González-Pastor JE: Cannibalism: a social behavior in sporulating Bacillus subtilis. FEMS microbiology reviews 2011, 35:415-424.

79. McCormick JR, Flärdh K: Signals and regulators that govern Streptomyces development. FEMS microbiology reviews 2012, 36:206-231.

80. Wireman JW, Dworkin M: Developmentally induced autolysis during fruiting body formation by Myxococcus xanthus. Journal of bacteriology 1977, 129:798-802.

81. Kessin RH: Dictyostelium: evolution, cell biology, and the development of multicellularity: Cambridge University Press; 2001.

82. Van Gestel J, Vlamakis H, Kolter R: Division of labor in biofilms: the ecology of cell differentiation. Microbiology spectrum 2015, 3:3.2. 26.

83. Zhang Z, Claessen D, Rozen DE: Understanding microbial divisions of labor. Frontiers in microbiology 2016, 7:2070.

84. Giri S, Waschina S, Kaleta C, Kost C: Defining division of labor in microbial communities. Journal of molecular biology 2019, 431:4712-4731.

85. Umen J, Herron MD: Green Algal Models for Multicellularity. Annual Review of Genetics 2021, 55.

86. Geerlings NM, Karman C, Trashin S, As KS, Kienhuis MV, Hidalgo-Martinez S, VasquezCardenas D, Boschker HT, De Wael K, Middelburg JJ: Division of labor and growth during electrical cooperation in multicellular cable bacteria. Proceedings of the National Academy of Sciences 2020, 117:5478-5485.

87. Zhang Z, Du C, de Barsy F, Liem M, Liakopoulos A, van Wezel GP, Choi YH, Claessen $D$, Rozen DE: Antibiotic production in Streptomyces is organized by a division of labor through terminal genomic differentiation. Science advances 2020, 6:eaay5781.

88. Kim W, Levy SB, Foster KR: Rapid radiation in bacteria leads to a division of labour. Nature communications 2016, 7:1-10.

89. Regenberg B, Hanghøj KE, Andersen KS, Boomsma JJ: Clonal yeast biofilms can reap competitive advantages through cell differentiation without being obligatorily multicellular. Proceedings of the Royal Society B: Biological Sciences 2016, 283:20161303.

90. Yanni D, Jacobeen S, Márquez-Zacarías P, Weitz JS, Ratcliff WC, Yunker PJ: Topological constraints in early multicellularity favor reproductive division of labor. Elife 2020, 9:e54348.

91. Airoldi L: Effects of disturbance, life histories, and overgrowth on coexistence of algal crusts and turfs. Ecology 2000, 81:798-814.

92. Boyce CK, Fan Y, Zwieniecki MA: Did trees grow up to the light, up to the wind, or down to the water? How modern high productivity colors perception of early plant evolution. New Phytologist 2017, 215:552-557.

93. Oliveira NM, Martinez-Garcia E, Xavier J, Durham WM, Kolter R, Kim W, Foster KR: Biofilm formation as a response to ecological competition. PLoS biology 2015, 13:e1002191.

94. Mitri S, Xavier JB, Foster KR: Social evolution in multispecies biofilms. Proceedings of the National Academy of Sciences 2011, 108:10839-10846.

95. Whiteside MD, Werner GD, Caldas VE, van't Padje A, Dupin SE, Elbers B, Bakker M, Wyatt GA, Klein M, Hink MA: Mycorrhizal fungi respond to resource inequality by 
moving phosphorus from rich to poor patches across networks. Current Biology 2019, 29:2043-2050. e2048.

96. Heaton LL, Jones NS, Fricker MD: A mechanistic explanation of the transition to simple multicellularity in fungi. Nature communications 2020, 11:1-9.

97. Graham LE: The occurrence, evolution, and phylogenetic significance of parenchyma in Coleochaete Bréb.(Chlorophyta). American Journal of Botany 1982, 69:447-454.

98. Umen JG: Green algae and the origins of multicellularity in the plant kingdom. Cold Spring Harbor Perspectives in Biology 2014, 6:a016170.

99. Reeve HK, Hölldobler B: The emergence of a superorganism through intergroup competition. Proceedings of the National Academy of Sciences 2007, 104:97369740.

100. Jarvis JU, O'Riain MJ, Bennett NC, Sherman PW: Mammalian eusociality: a family affair. Trends in Ecology \& Evolution 1994, 9:47-51.

101. Solari CA, Ganguly S, Kessler JO, Michod RE, Goldstein RE: Multicellularity and the functional interdependence of motility and molecular transport. Proceedings of the National Academy of Sciences 2006, 103:1353-1358.

102. Claessen D, Rozen DE, Kuipers OP, Søgaard-Andersen L, Van Wezel GP: Bacterial solutions to multicellularity: a tale of biofilms, filaments and fruiting bodies. Nature Reviews Microbiology 2014, 12:115-124.

103. Raper KB: The dictyostelids, vol 561: Princeton University Press; 2014.

104. Lawal HM, Schilde C, Kin K, Brown MW, James J, Prescott AR, Schaap P: Cold climate adaptation is a plausible cause for evolution of multicellular sporulation in Dictyostelia. Scientific reports 2020, 10:1-9.

105. Ward MJ, Zusman DR: Developmental aggregation and fruiting body formation in the gliding bacterium Myxococcus xanthus. Prokaryotic Development 1999:243262.

106. Bonner J: Evolutionary strategies and developmental constraints in the cellular slime molds. The American Naturalist 1982, 119:530-552.

107. Kaiser D: Building a multicellular organism. Annual review of genetics 2001, 35:103123.

108. Queller DC, Strassmann JE: Fruiting bodies of the social amoeba Dictyostelium discoideum increase spore transport by Drosophila. BMC evolutionary biology 2014, 14:1-5.

109. Bonner J, Lamont D: Behavior of cellular slime molds in the soil. Mycologia 2005, 97:178-184.

110. Velicer GJ, Vos M: Sociobiology of the myxobacteria. Annual review of microbiology 2009, 63:599-623.

111. Toda S, Blauch LR, Tang SK, Morsut L, Lim WA: Programming self-organizing multicellular structures with synthetic cell-cell signaling. Science 2018, 361:156162.

112. Hammerschmidt K, Rose CJ, Kerr B, Rainey PB: Life cycles, fitness decoupling and the evolution of multicellularity. Nature 2014, 515:75-79. 